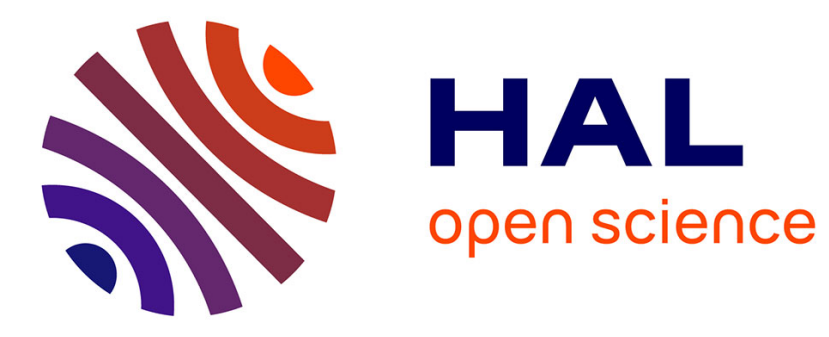

\title{
Algorithms for Outsourcing Pairing Computation
}

\author{
Aurore Guillevic, Damien Vergnaud
}

\section{To cite this version:}

Aurore Guillevic, Damien Vergnaud. Algorithms for Outsourcing Pairing Computation. CARDIS 2014 - 13th Smart Card Research and Advanced Application Conference, Conservatoire National des Arts et Métiers (CNAM), Nov 2014, Paris, France. 10.1007/978-3-319-16763-3_12 . hal-01084550

\section{HAL Id: hal-01084550 \\ https://hal.inria.fr/hal-01084550}

Submitted on 13 May 2020

HAL is a multi-disciplinary open access archive for the deposit and dissemination of scientific research documents, whether they are published or not. The documents may come from teaching and research institutions in France or abroad, or from public or private research centers.
L'archive ouverte pluridisciplinaire HAL, est destinée au dépôt et à la diffusion de documents scientifiques de niveau recherche, publiés ou non, émanant des établissements d'enseignement et de recherche français ou étrangers, des laboratoires publics ou privés. 


\title{
Algorithms for Outsourcing Pairing Computation
}

\author{
Aurore Guillevic $^{2,3}$ and Damien Vergnaud ${ }^{1}$ \\ 1 Département d'Informatique, École normale supérieure, Paris, France \\ 2 Inria, France \\ 3 École Polytechnique/LIX, France
}

\begin{abstract}
We address the question of how a computationally limited device may outsource pairing computation in cryptography to another, potentially malicious, but much more computationally powerful device. We introduce two new efficient protocols for securely outsourcing pairing computations to an untrusted helper. The first generic scheme is proven computationally secure (and can be proven statistically secure at the expense of worse performance). It allows various communication-efficiency trade-offs. The second specific scheme - for optimal Ate pairing on a Barreto-Naehrig curve - is unconditionally secure, and do not rely on any hardness assumptions. Both protocols are more efficient than the actual computation of the pairing by the restricted device and in particular they are more efficient than all previous proposals.
\end{abstract}

\section{Introduction}

Pairings (or bilinear maps) were introduced in cryptography in 2000 by Joux [14] and Boneh-Franklin [4]. A pairing is a bilinear, non-degenerate and computable map $e: \mathbb{G}_{1} \times \mathbb{G}_{2} \rightarrow \mathbb{G}_{T}$. In practice, the first two groups $\mathbb{G}_{1}$ and $\mathbb{G}_{2}$ are prime-order $r$ subgroups of the group of points $E\left(\mathbb{F}_{q}\right)$ of an elliptic curve $E$ defined over a finite field $\mathbb{F}_{q}$. The so-called target group $\mathbb{G}_{T}$ is the order $r$ subgroup of a finite field extension $\mathbb{F}_{q^{k}}$. Bilinear pairings proved to be an amazingly flexible and useful tool for the construction of cryptosystems with unique features (e.g. efficient identity based cryptography or short signatures). However, the pairing computation is more resource consuming compared to a scalar multiplication on the elliptic curve $E\left(\mathbb{F}_{q}\right)$.

In the last decade, several papers [12]9|7] studied the question of how a computationally limited device may outsource pairing computation to another, potentially malicious, but much more computationally powerful device. In this setting, one wants to efficiently delegate the computation of a pairing $e\left(\mathcal{S} \mathcal{K}_{1}, \mathcal{S} \mathcal{K}_{2}\right)$ of two secret keys, or a pairing $e(\mathcal{S K}, \mathcal{P} \mathcal{P})$ of a secret key and some public parameter. Obviously, one needs to ensure that this malicious device does not learn anything about what it is actually computing (secrecy) and sometimes one also needs to, when possible, detect any failures (verifiability, also called correctness).

As mentioned in [977, a delegation protocol that does not ensure verifiability may cause severe security problems (in particular if the pairing computation occurs in the verification algorithm of some authentication protocol). Unfortunately, the different proposals for verifiable pairing delegation are very inefficient and it is actually better in practice to directly embed the pairing computation inside the restricted device 
than using these solutions. The main interest is then to save of area that is required to implement a pairing in the restricted device such as smart card.

However, if verifiability is mandatory in authentication protocols, this is not necessarily the case in scenarios where the delegated pairing value is used in an encryption scheme as a session key. In this case, one can indeed use additional cryptographic techniques to ensure that the values returned by the powerful device are correct (e.g. by adding a MAC or other redundancy to the ciphertext). One can even consider settings where the powerful device actually learns the pairing value. For instance, in a pay-TV scenario, the set-up box (provided by the pay-TV company) needs to know the (onetime) session key $\mathcal{K}$ used to decipher the content (e.g. movie, football match) but it does not know the secret key $\mathcal{S K}$ securely stored in the smartcard. If the smartcard delegates the pairing computation to the set-up box there is no harm to let it know the session key $\mathcal{K}$ since it will learn it anyway.

In 2005, Girault and Lefranc [12] introduced the first secure pairing delegation protocol through the Server-Aided Verification notion which consists in speeding up the verification step of an authentication/ signature scheme. Their pairing delegation protocol only achieves secrecy with unconditional security (and the verifiability is achieved via a different mean). Chevallier-Mames, Coron, McCullagh, Naccache and Scott introduced in 2005 the security notions for pairing delegation [8|9] and they provided a verifiable delegation protocol for pairing computation. Their protocols are much more resource consuming for the restricted device than directly computing the pairing.

Recently, Canard, Devigne and Sanders proposed a more efficient protocol for verifiable pairing delegation. The authors showed that their proposal is more efficient than the computation of the pairing for optimal ate pairing on a so-called KSS-18 curve [15]. Unfortunately, we will show in this paper that this is not the case for state-of-the-art optimal Ate pairing on a Barreto-Naehrig curve [3].

Contributions of the paper. We propose two new efficient protocols for secret pairing delegation. Our protocols are not verifiable but as explained above, this is not really an issue for encryption primitives where verifiability can be achieved by other means. In particular, our typical usecases are Pay-TV where a smartcard delegates a pairing computation to the set-up box and encrypted GSM communication where the sim-card delegates a pairing computation to the smartphone processor (e.g. an ARM or Intel processor with high competitive performances). In these scenarios, one can even assume that the set-up box or the smartphone actually learns the pairing value (but of course not the secret information stored by the smartcard or the sim-card). Both methods enable to delegate the computation of a pairing $e(\mathcal{S K}, \mathcal{P} \mathcal{P})$ of a secret key $\mathcal{S K}$ and some public parameter $\mathcal{P} \mathcal{P}$. They achieve better efficiency than actual computation of the pairing by the restricted device and in particular they are more efficient than all previous proposals.

We first present a (generalized) knapsack-based approach which uses different endomorphisms on the groups $\mathbb{G}_{1}, \mathbb{G}_{2}, \mathbb{G}_{T}$ to speed-up the method. Instead of masking the secret point $\mathcal{S K}$ by a scalar multiplication with a random secret exponent, it is masked by adding to it a sum of (small) multiple of random points that are also send to the powerful device. It computes several pairings of these points with the public parameter $\mathcal{P} \mathcal{P}$ and the restricted device combines them to get the actual value. The 
method is generic and can be applied to any pairing instantiation. The method increases the communication complexity between the two devices but one can propose different communication-efficiency trade-off.

In our second approach, we present a way to delegate only the non-critical steps in the pairing algorithm, looking carefully at each instruction in Miller algorithm. The powerful device does not learn any information on the secret point $\mathcal{S K}$ except the actual value of the pairing $e(\mathcal{S K}, \mathcal{P} \mathcal{P})$ (which is perfectly suitable in our usecases). The technique can be applied to any instantiation of pairings but we concentrate on the state-of-the-art optimal Ate pairing on a Barreto-Naehrig curve [3]. We obtain a $65 \%$ improvement (for a 128-bit security level) for the restricted device compared to the computation of the pairing.

\section{Preliminaries}

Timing Estimates using the Relic Library. To illustrate the algorithms presented in this paper, we estimate the various costs of scalar multiplication, exponentiations and pairings. We choose as a practical example a Barreto-Naehrig (BN) curve [3] at the 128-bit security level with the implementation provided in Relic library of Aranha [1].

This library is at the state of the art for pairing computation [2] and is freely available for research purpose. We assume that scalar multiplications $[a] P$ and exponentiations $z^{a}$ are performed with a binary signed representation of $a$. So it requires roughly $\log a$ doublings (resp. squarings) and $\log a / 3$ additions (resp. multiplications). A doubling on a BN curve (with $a_{4}=0$ ) costs $2 M_{p}+5 S_{p}$ (multiplications and squarings in a finite field $\mathbb{F}_{p}$ ) and a mixed addition costs $7 M_{p}+4 S_{p}$ [1]. We assume that $M_{p}=S_{p}$ first because it is the case for Relic library and secondly to simplify (but the estimation $S_{p}=0.9 M_{p}$ would also be accurate for another implementation). We obtain a total cost of $\approx 256 \mathrm{Dbl}_{E\left(\mathbb{F}_{p}\right)}+86 \operatorname{Add}_{E\left(\mathbb{F}_{p}\right)} \approx 2738 M_{p}$ for a scalar multiplication on $\mathbb{G}_{1}, 2.4$ times this cost: $\approx 6590 M_{p}$ for a scalar multiplication in $\mathbb{G}_{2}$ and $\approx 256 S_{p^{12}}+86 M_{p^{12}} \approx$ $9252 M_{p}$ for an exponentiation in $\mathbb{G}_{T}$. We note that checking that an element is in $\mathbb{G}_{T}$ is much more expensive than performing an exponentiation in $\mathbb{G}_{T}$. Indeed $\mathbb{G}_{T}$ is an order$r$ subgroup in a large finite field $\mathbb{F}_{p^{k}}$. $\mathbb{G}_{T}$ has particular properties permitting very fast squaring that $\mathbb{F}_{p^{k}}$ does not. We summarize these estimates in Tab. 1] (which may be of independent interest).

Optimal Ate pairing on a Barreto-Naehrig Curve A pairing is computed in two steps (see Alg. 11: a Miller function $f \leftarrow f_{r, Q}(P)$ (Alg. 1. lines 1-15) followed by a

final powering $f^{\frac{p^{k}-1}{r}}$ (Alg.1 lines $16-27$ ) to obtain a unique value in $\mathbb{G}_{T}$, the subgroup of order $r$ of $\mathbb{F}_{p^{*}}^{*}$.

There are several papers on pairing computation on $\mathrm{BN}$ curves. We present in Alg.1 all the steps for an optimal ate pairing computation on a BN curve. Our global estimate is $16336 M_{p}$ (multiplications in $\mathbb{F}_{p}$ ) for one pairing. The Miller loop takes $8425 M_{p}$ (52\%) and the exponentation $7911 M_{p}(48 \%)$. From Relic benchmarks on an Intel Xeon CPU E5-1603 0 at $2.8 \mathrm{GHz}$, we obtain one pairing in $3.241 \mathrm{~ms}$, the Miller loop in $1.776 \mathrm{~ms}(55 \%)$ and the exponentiation in $1.465 \mathrm{~ms}(45 \%)$. 
Table 1. Estimations for common operations in algorithms, for a BN curve with $\log p=$ 256 bits and Relic [1] implementation (Running Relic toolkit on a Intel Xeon E5-1603 at $2.80 \mathrm{GHz}$ ).

\begin{tabular}{|c|c|c|c|}
\hline Operation & $\cos t$ & total over $\mathbb{F}_{p}$ & Relic \\
\hline \multicolumn{4}{|c|}{$\mathbb{F}_{p^{k}}$ arithmetic } \\
\hline & & $M_{p}$ & $0.149 \mu \mathrm{s}$ \\
\hline$M_{p^{2}}$ & $3 M_{p}$ & $3 M_{p}$ & $0.427 \mu \mathrm{s}$ \\
\hline$S_{p^{2}}$ & $2 M_{p}$ & $2 M_{p}$ & $0.360 \mu \mathrm{s}$ \\
\hline$M_{p^{6}}$ & $6 M_{p^{2}}$ & $18 M_{p}$ & $3.362 \mu \mathrm{s}$ \\
\hline$S_{p^{6}}$ & $2 M_{p^{2}}+3 S_{p^{2}}$ & $12 M_{p}$ & $2.523 \mu \mathrm{s}$ \\
\hline$M_{p^{12}}$ & $3 M_{p^{6}}$ & $54 M_{p}$ & $10.856 \mu \mathrm{s}$ \\
\hline$S_{p^{12}}$ & $2 M_{p^{6}}$ & $36 M_{p}$ & $7.598 \mu \mathrm{s}$ \\
\hline$S_{\phi_{12}(p)}$ & $z^{2}, z \in \mathbb{F}_{p^{12}}, \operatorname{Norm}(z)=1$ & $18 M_{p}$ & $4.731 \mu \mathrm{s}$ \\
\hline$z^{a}$, for any $z, a$ & $\log a S_{p^{12}}+\log a / 3 M_{p^{12}}$ & $54 \log a M_{p}$ & $3.864 \mathrm{~ms}$ \\
\hline$z^{a}, \operatorname{Norm}_{\mathbb{F}_{p} 12} / \mathbb{F}_{p}(z)=1$ & $\log a S_{\phi_{12}(p)}+\log a / 3 M_{p^{12}}$ & $36 \log a M_{p}$ & $2.818 \mathrm{~ms}$ \\
\hline $\operatorname{Norm}_{\mathbb{F}_{p} 12} / \mathbb{F}_{p}(z)$, for any $z \mid$ & 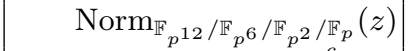 & $59 M_{p}$ & - \\
\hline$z^{r}, \operatorname{Norm}_{\mathbb{F}_{p^{12}} / \mathbb{F}_{p}}(z)=1$ & $z^{p} z^{1-t}=z^{p}\left(z^{p^{6}}\right)^{t-1}$ & $4616 M_{p}$ & - \\
\hline check order $(z)=r$ in $\mathbb{F}_{p^{k}}$ & $\operatorname{Norm}_{\mathbb{F}_{p} 12} / \mathbb{F}_{p}(z)=1 ; z^{r}=1$ & $4675 M_{p}$ & - \\
\hline \multicolumn{4}{|c|}{$E\left(\mathbb{F}_{p}\right)$ arithmetic } \\
\hline$\left(\mathrm{Dbl}_{p}\right)$ & $2 M_{p}+5 S_{p}$ & $7 M_{p}$ & $1.043 \mu \mathrm{s}$ \\
\hline Addition $\left(\operatorname{Add}_{p}\right)$ & $7 M_{p}+4 S_{p}$ & $11 M_{p}$ & $1.639 \mu \mathrm{s}$ \\
\hline Scalar mult. $[a] P$ & $\log a \mathrm{Dbl}+\log a / 3 \mathrm{Add}$ & $10.7 \log a M_{p}$ & - \\
\hline$\left[a_{1}\right] P_{1}+\left[a_{2}\right] P_{2}$ & $\max \left(\log a_{1}, \log a_{2}\right)$ & $\max \left(\log a_{1}, \log a_{2}\right)$ & \\
\hline $\mid\left[\omega_{1}\right] I_{1}+\left[\omega_{2}\right] I_{2}$ & $(\mathrm{Dbl}+2 / 3 \mathrm{Add})$ & $14.33 M_{p}$ & \\
\hline \multicolumn{4}{|c|}{$E\left(\mathbb{F}_{p^{2}}\right)$ arithmetic } \\
\hline Doubling $\left(\mathrm{Dbl}_{p^{2}}\right)$ & $2 M_{p^{2}}+5 S_{p^{2}}$ & $16 M_{p}$ & $3.137 \mu \mathrm{s}$ \\
\hline Addition $\left(\operatorname{Add}_{p^{2}}\right)$ & $7 M_{p^{2}}+4 S_{p^{2}}$ & $29 M_{p}$ & $4.866 \mu \mathrm{s}$ \\
\hline Scalar mult. $[b] Q$ & $\log b \mathrm{Dbl}_{p^{2}}+\log b / 3 \mathrm{Add}_{p^{2}}$ & $25.7 \log b M_{p}$ & $2.017 \mathrm{~ms}$ \\
\hline$\left[b_{1}\right] Q_{1}+\left[b_{2}\right] Q_{2}$ & $\max \left(\log b_{1}, \log b_{2}\right)$ & $\max \left(\log b_{1}, \log b_{2}\right)$ & \\
\hline \multicolumn{4}{|c|}{ Pairing on a BN curve with $\log _{2} p=256$} \\
\hline Dbl step $+\ell_{T, T}(P)$ & $3 M_{p^{2}}+7 S_{p^{2}}+4 M_{p}$ & $27 M_{p}$ & $6.036 \mu \mathrm{s}$ \\
\hline Add step $+\ell_{T, Q}(P)$ & $11 M_{p^{2}}+2 S_{p^{2}}+4 M_{p}$ & $41 M_{p}$ & $7.593 \mu \mathrm{s}$ \\
\hline Miller loop & see Alg. 1 & $8425 M_{p}$ & $1.776 \mathrm{~ms}$ \\
\hline Final powering & see Alg. 1 & $7911 M_{p}$ & $1.465 \mathrm{~ms}$ \\
\hline Pairing & see $A \lg .1$ & $16336 M_{p}$ & $3.241 \mathrm{~ms}$ \\
\hline
\end{tabular}

Security Model for Pairing Delegation In this subsection, we provide an informal description of the security model for pairing delegation protocol and refer the reader to the papers [9]7 for more details. We consider only protocols for delegation of a pairing $e(\mathcal{S K}, \mathcal{P} \mathcal{P})$ of a secret key $\mathcal{S K}$ and some public parameter $\mathcal{P} \mathcal{P}$. The security notions defined in [9]7] are the following:

Secrecy requires that the powerful device cannot learn any information on $\mathcal{S} \mathcal{K}$. 


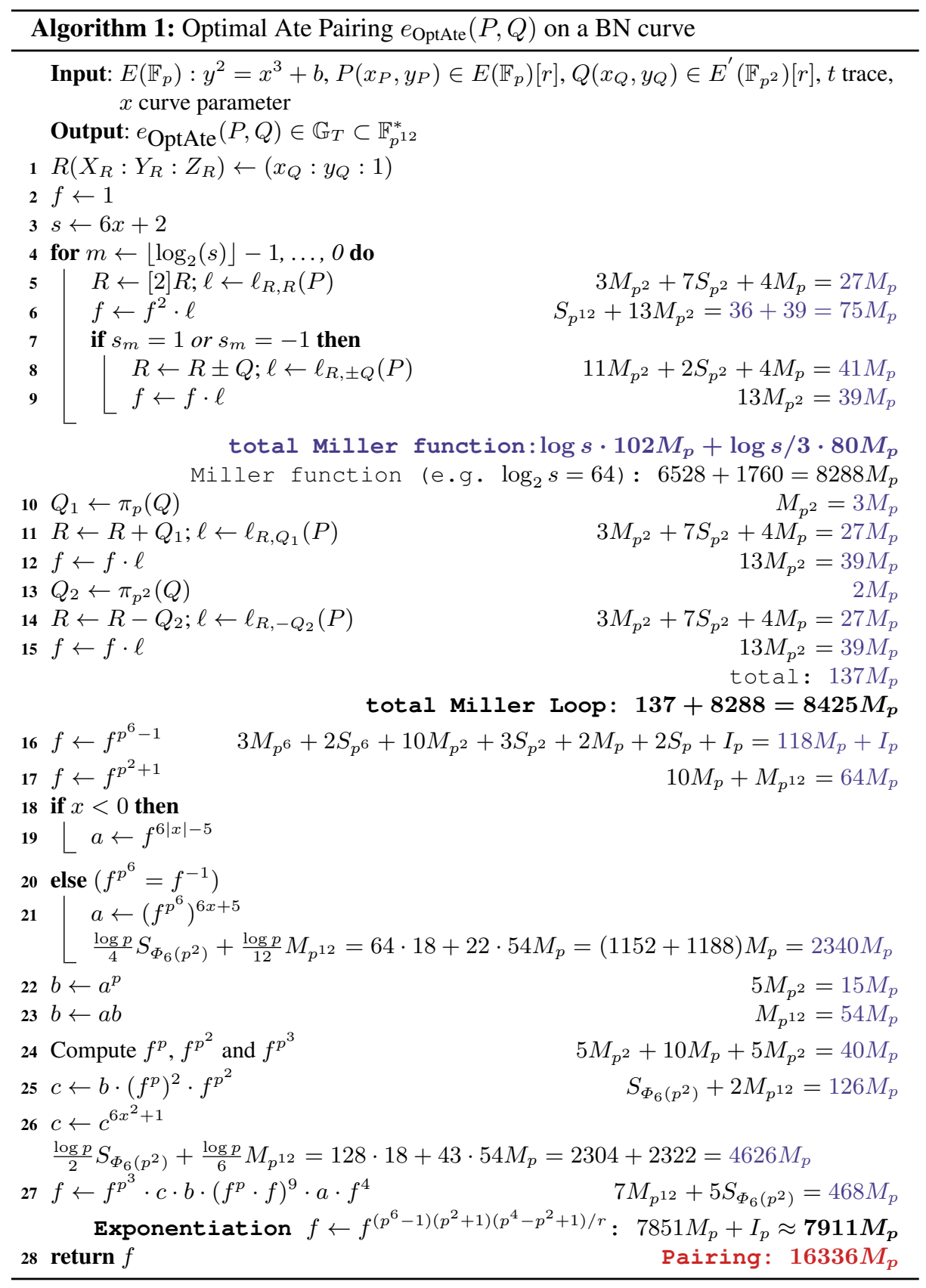

Verifiability requires that the restricted device, even interacting with a dishonest powerful device, will not output a wrong value for $e(\mathcal{S K}, \mathcal{P} \mathcal{P})$. 
The formal security game for secrecy is similar to the indistinguishability security notion for encryption schemes. The adversary chooses two secret points $\mathcal{S} \mathcal{K}_{0}$ and $\mathcal{S K}_{1}$ and runs the delegation protocol with the restricted device for the secret point $\mathcal{S} \mathcal{K}_{b}$ for some bit $b$. The scheme achieves secrecy if the probability that a (polynomial-time) adversary guesses the bit $b$ is negligibly close to $1 / 2$. The formal security game for verifiability ensures that at the end of the delegation protocol, the restricted device obtains the actual value $e(\mathcal{S K}, \mathcal{P} \mathcal{P})$ or knows that the powerful device cheated in the protocol.

As mentioned above, in some cases, the secrecy property is too strong if the powerful device is allowed to learn the value $e(\mathcal{S K}, \mathcal{P} \mathcal{P})$ afterwards. Indeed this value reveals some information on $\mathcal{S K}$ and the complete protocol does not achieve the secrecy property. Therefore, we propose the following notion which is weaker than the secrecy notion but well-suited for our usecases of Pay-TV and encrypted GSM communication:

Weak Secrecy requires that the powerful device cannot learn any information about $\mathcal{S K}$ except what can be deduced from the value $e(\mathcal{S K}, \mathcal{P} \mathcal{P})$

Let us assume that we use a pairing delegation protocol for the decryption in a pairingbased scheme (such as the well-known Boneh-Franklin identity-based encryption [4]). If the delegation protocol achieves only Weak Secrecy, a malicious powerful device can mount a lunch-time attack (or CCA1) against the encryption scheme (using the restricted device in the delegation protocol as a decryption oracle). However, since it does not learn any information about $\mathcal{S K}$ (except from the one-time session keys $e\left(\mathcal{S K}, \mathcal{P} \mathcal{P}_{i}\right)$ for several public parameters $\mathcal{P} \mathcal{P}_{i}$ 's), it is not able to decrypt any ciphertext if the restricted device is no longer active (e.g. after revocation of the decryption rights in the Pay-TV scenario).

\section{Review of Previous Proposals}

\subsection{Girault-Lefranc Pairing Delegation Protocol}

In this subsection, we present Girault-Lefranc protocol for server-aided signature verification [12, §4.1] in Alg. 2 with a performance estimation on a BN curve at a 128-bit security level ( $\log r=\log p=256)$ using the Relic library described above.

Our cost estimation concludes that the delegation of $e(\mathcal{S K}, \mathcal{S P})$ with secret $\mathcal{S K}, \mathcal{S P}$ costs $\approx 18640 M_{p}$ which is more than a pairing computation at the state of the art (we estimate this for $16336 M_{p}$ in Relic library).

Note that if pre-computation is possible, then the computation of $[a] \mathcal{S K}$ in the first step of Alg. 2 can actually be done off-line. If moreover, the point $\mathcal{S P}$ is public, then the complexity of the delegation protocol falls down to $9252 M_{p}$ (i.e. 0.6 pairing). This basic scheme (with pre-computation) is the most efficient pairing delegation protocol (without verifiability) of a pairing $e(\mathcal{S K}, \mathcal{P} \mathcal{P})$ of a secret key $\mathcal{S K}$ and some public parameter $\mathcal{P} \mathcal{P}$.

In Girault-Lefranc delegation, as $f$ is a pairing output, we can use the optimized squaring formula of Granger and Scott [13] when computing $f^{(a b)^{-1}}$, hence $S_{p^{12}}=$ $18 M_{p}$ instead of $36 M_{p}$. The computations over the group $\mathbb{G}_{1}$ might be available on the restricted device such as a smartcard. More precisely, we need multiplication $\left(M_{p}\right)$, addition - subtraction $\left(A_{p}\right)$ and inversion $I_{p}$ in $\mathbb{F}_{p}$. Finite field operations are implemented 


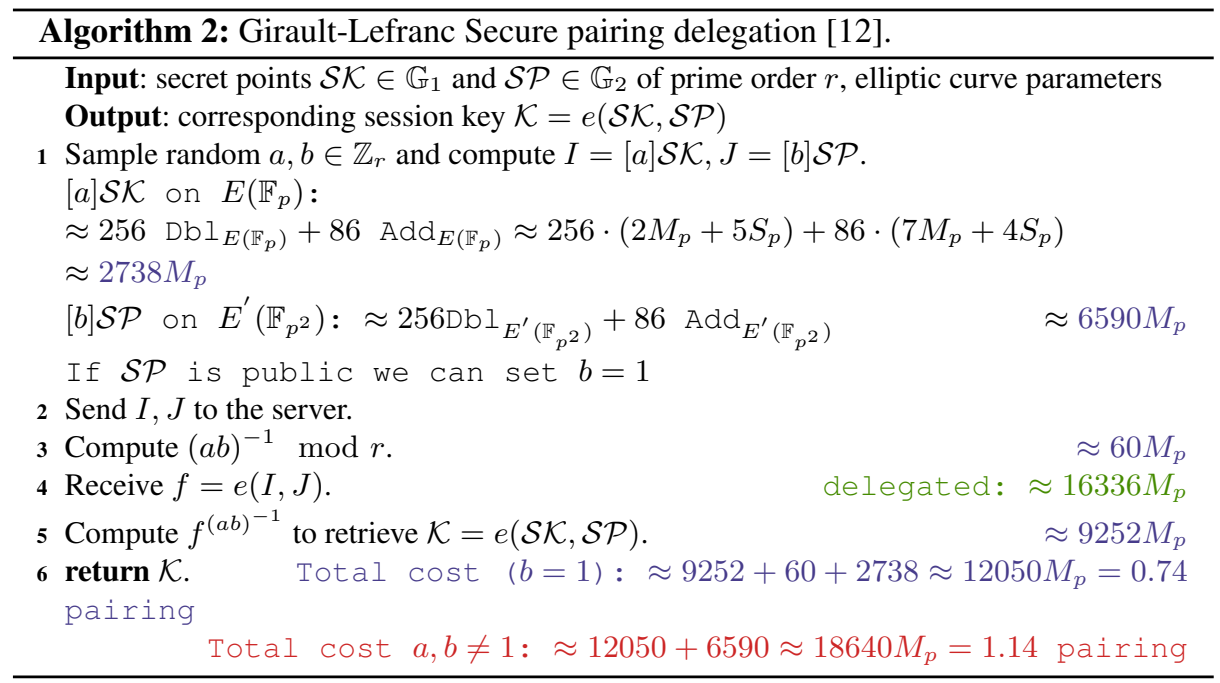

on a smartcard e.g. for ECDSA but the arithmetic operations are not available for the user. We can use the RSA primitives to simulate $\mathbb{F}_{p}$ arithmetic. We set no padding, the exponent to 2 and the "RSA modulus" to $p$ to get squares $\bmod p$, then simulate multiplications through $2 x y=(x+y)^{2}-x^{2}-y^{2}$. Computations in the group $\mathbb{G}_{T}$ are not available and must be implemented. If a BN curve [3] is used, $\mathbb{G}_{T} \subset \mathbb{F}_{p^{12}}^{*}$ hence a complicated arithmetic must be implemented.

Remark 1 (Lightening the Girault-Lefranc scheme). If the session key $\mathcal{K}$ can be known by the untrusted helper (i.e. if one only needs weak secrecy), we note that a variant of the protocol may be used in some cases. We propose to ask the external resource to compute $e\left([\alpha] \mathcal{S K},\left[\alpha^{-1}\right] \mathcal{S P}\right)=e(\mathcal{S K}, \mathcal{S P})=\mathcal{K}$ with $\alpha$ taken at random. The output will be exactly $\mathcal{K}$. This solution is not very efficient as it costs $9388 / 16336=0.6$ pairing. To improve it slightly in practice, we can swap $\mathcal{S K}$ and $\mathcal{P} \mathcal{P}$, i.e. put $\mathcal{S K}$ in $E^{\prime}\left(\mathbb{F}_{p^{2}}\right)$ and $\mathcal{P P} \in E\left(\mathbb{F}_{p}\right)$. In this way, $[\alpha] \mathcal{S K}$ is the costly part and can be computed offline. Note that this delegation procedure reveals some information on the secret key $\mathcal{S K}$ and it is necessary to reprove the security of the underlying scheme if it is used to improve its efficiency.

\subsection{Chevallier-Mames et al. Pairing Delegation Protocol}

Another pairing delegation protocol was introduced by Chevallier-Mames, Coron, McCullagh, Naccache and Scott in 2005 [8]9]. Contrary to Girault-Lefranc's protocol, the protocol proposed by Chevallier-Mames et al. achieves secrecy (unconditionnally) and verifiability. Unfortunately, the protocol is very inefficient since the overall cost for the restricted device is 3.5 times the cost for computing the pairing ( 3.3 if pre-computation is possible). The main advantage of the scheme is to save of area that is required to implement a pairing in the restricted device such a smart card. However, as mentioned 
above, even if we can use tricks, computations in the group $\mathbb{G}_{T}$ are usually not available and must be implemented (i.e. complex arithmetic in $\mathbb{G}_{T} \subset \mathbb{F}_{p^{12}}^{*}$ for a BN curve).

\subsection{Canard-Devigne-Sanders Pairing Delegation Protocol}

We present in Alg. 3 the pairing delegation protocol proposed recently by Canard, Devigne and Sanders [7]. The protocol is more efficient than the previous one. It also achieves secrecy (unconditionnally) and verifiability. Canard et al. actually showed that their proposal is in fact more efficient than the computation of the pairing for optimal ate pairing on a so-called KSS-18 curve [15]. Unfortunately, as shown by the precise complexity of Alg. 3, this is not the case for state-of-the-art optimal Ate pairing on a BN curve [3]. More precisely, we show that the overall cost for the restricted device is 2.8 times the cost for computing the pairing (1.6 if pre-computation is possible).

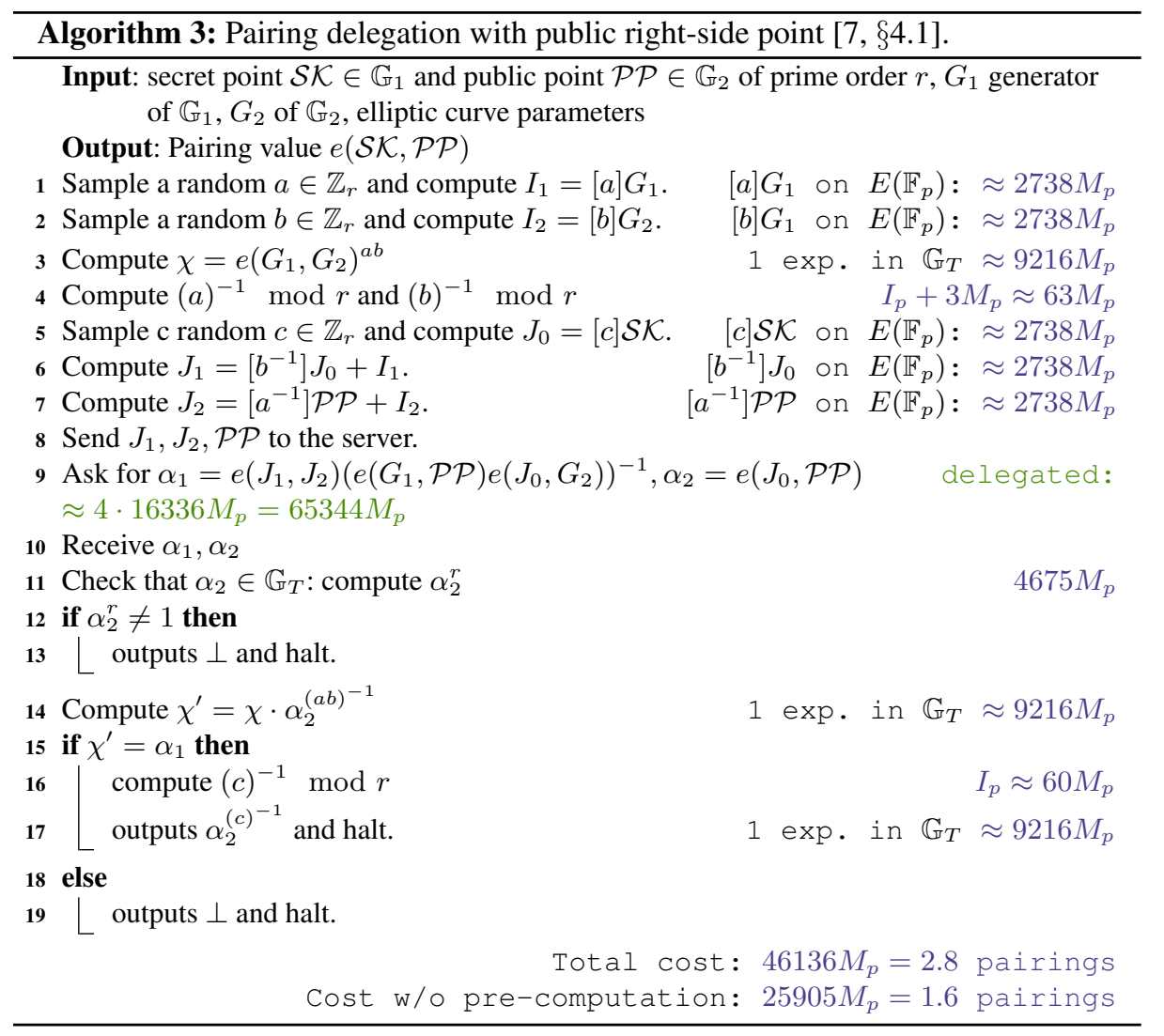




\section{Pairing Delegation with Knapsack}

We present in this section a new approach to perform pairing delegation (without verifiability) of a pairing $e(\mathcal{S} \mathcal{K}, \mathcal{P} \mathcal{P})$ of a secret key $\mathcal{S K}$ and some public parameter $\mathcal{P} \mathcal{P}$. The restricted device (e.g. a smartcard) generates random points and sends them to the powerful device to compute several pairings. The smartcard receives the pairings and combines some of them to get the actual value $e(\mathcal{S} \mathcal{K}, \mathcal{P} \mathcal{P})$. The basic idea is to mask the secret value $\mathcal{S K}$ by a linear combination of those random points with "small" coefficients to improve efficiency. A similar approach has been used successfully in the setting of server-aided exponentiation [6 16].

\subsection{Security Analysis}

Let $\mathbb{G}$ be a cyclic group of order $p$ denoted additively. We consider the two following distributions:

$$
\mathcal{U}_{n}=\left\{\left(P_{1}, P_{2}, \ldots, P_{n}, Q\right) \stackrel{R}{\longleftarrow} \mathbb{G}^{n+1}\right\}
$$

and

$$
\mathcal{K}_{n, A}=\left\{\begin{array}{c}
\left(P_{1}, P_{2}, \ldots, P_{n}\right) \stackrel{R}{\longleftarrow} \mathbb{G}^{n} \\
\left(P_{1}, P_{2}, \ldots, P_{n}, Q\right), \text { s.t. } Q \leftarrow\left[a_{1}\right] P_{1}+\cdots+\left[a_{n}\right] P_{n} \\
\text { where }\left(a_{1}, \ldots, a_{n}\right) \stackrel{R}{\longleftarrow} \llbracket 0, A-1 \rrbracket^{n}
\end{array}\right\} .
$$

$\mathcal{U}_{n}$ is the uniform distribution on $\mathbb{G}^{n+1}$ and $\mathcal{K}_{n, A}$ outputs $(n+1)$-tuples where the first $n$ components are picked uniformly at random in $\mathbb{G}$ while the last component is a linear combination of those elements with exponents picked uniformly at random in the interval $\llbracket 0, A-1 \rrbracket$. In a basic version of our delegation protocol, the restricted device sends the elements $\left(P_{1}, \ldots, P_{n}\right)$ and $P_{n+1}=(\mathcal{S K}-Q)$ to the powerful device. It replies by sending back the pairings $e\left(P_{i}, \mathcal{P} \mathcal{P}\right)$ for $i \in\{1, \ldots, n+1\}$. The restricted device finally gets $e(\mathcal{S} \mathcal{K}, \mathcal{P} \mathcal{P})$ as $e\left(P_{n+1}, \mathcal{P} \mathcal{P}\right) \cdot \prod_{i=1}^{n} e\left(g_{i}, \mathcal{P} \mathcal{P}\right)^{a_{i}}$. If the two distributions $\mathcal{U}_{n}$ and $\mathcal{K}_{n, A}$ are indistinguishable, the protocol will readily achieve the secrecy property.

- Perfect indistinguishability. It is straightforward to see that if $A=p$, then the two distributions are identical (even if $n=1$ ) and the delegation scheme as outlined above achieves unconditional secrecy. Unfortunately, as we will see in the next paragraph, the efficiency of our schemes depends crucially on the size of $A$ and one wants to use smaller $A$ in practice.

- Statistical indistinguishability. By using classical results on the distribution of modular sums [16], one can prove that if $A^{n}=\Omega\left(p^{2}\right)$, then the two distributions $\mathcal{U}_{n}$ and $\mathcal{K}_{n, A}$ are statistically indistinguishable (see [1610] for details). For these parameters, the delegation protocol achieves statistical (and therefore computational) secrecy. For cryptographic purposes, the order $p$ of $\mathbb{G}$ needs to be of $2 k$-bit size to achieve a $k$-bit security level. Therefore, to achieve statistical indistinguishability, we need to have $A^{n}=\Omega\left(2^{4 k}\right)$ and the resulting delegation protocol is not really efficient. 
- Computational indistinguishability. For smaller parameters (i.e. $A^{n}=o\left(p^{2}\right)$ ), we cannot prove that the $\mathcal{U}_{n}$ and $\mathcal{K}_{n, A}$ are statistically indistinguishable. However, it may be possible to prove that they are computationally indistinguishable. Using a variant of Shanks "baby-step giant-step" algorithm, one can see easily that it is possible to find the scalars $\left(a_{1}, \ldots, a_{n}\right)$ (if they exist) such that $Q=\left[a_{1}\right] P_{1}+\cdots+$ $\left[a_{n}\right] P_{n}$ in $O\left(A^{n / 2}\right)$ group operations in $\mathbb{G}$ (i.e. to solve the generalized knapsack problem in $\mathbb{G}$ ). Therefore, to achieve computational indistinguishability for a $k$-bit security parameter, one needs to have at least $A^{n}=\Omega\left(2^{2 k}\right)=\Omega(p)$.

To conclude this paragraph, we will prove that the two distributions $\mathcal{U}_{n}$ and $\mathcal{K}_{n, A}$ are computationally indistinguishable in the generic group model when $A^{n}=\Omega\left(2^{2 k}\right)=$ $\Omega(p)$. Our delegation protocol therefore achieves secrecy in the generic group model when $A^{n}=\Omega\left(2^{2 k}\right)=\Omega(p)$. This model was introduced by Shoup [17] for measuring the exact difficulty of solving discrete logarithm problems. Algorithms in generic groups do not exploit any properties of the encodings of group elements. They can access group elements only via a random encoding algorithm that encodes group elements as random bit-strings.

Let $\mathcal{A}$ be a generic group adversary. As usual, the generic group model is implemented by choosing a random encoding $\sigma: \mathbb{G} \longrightarrow\{0,1\}^{m}$. Instead of working directly with group elements, $\mathcal{A}$ takes as input their image under $\sigma$. This way, all $\mathcal{A}$ can test is string equality. $\mathcal{A}$ is also given access to an oracle computing group addition and subtraction: taking $\sigma\left(R_{1}\right)$ and $\sigma\left(R_{2}\right)$ and returning $\sigma\left(R_{1}+R_{2}\right)$, similarly for subtraction. Finally, we can assume that $\mathcal{A}$ submits to the oracle only encodings of elements it had previously received. This is because we can choose $m$ large enough so that the probability of choosing a string that is also in the image of $\sigma$ is negligible.

Theorem 1. Let $\mathcal{A}$ be a generic algorithm that distinguishes the two distributions $\mathcal{U}_{n}$ and $\mathcal{K}_{n, A}$ that makes at most $\tau$ group oracle queries, then $\mathcal{A}$ 's advantage in distinguishing the two distributions is upper-bounded by $O\left(\tau^{2} / A^{n}\right)$.

To prove this theorem, we consider the following distributions in a product group $\mathbb{G}_{1} \times \cdots \times \mathbb{G}_{n}$ where each $\mathbb{G}_{i}$ is cyclic group of prime order $p$ (for $i \in\{1, \ldots, n\}$ ).

$$
\mathcal{U}_{n}^{\prime}=\left\{\left(P_{1}, P_{2}, \ldots, P_{n}, Q\right) \stackrel{R}{\longleftarrow} \mathbb{G}_{1} \times \mathbb{G}_{2} \times \cdots \times \mathbb{G}_{n} \times\left(\mathbb{G}_{1} \times \mathbb{G}_{2} \times \cdots \times \mathbb{G}_{n}\right)\right\}
$$

and

$$
\mathcal{K}_{n, A}^{\prime}=\left\{\begin{array}{c}
\left(P_{1}, P_{2}, \ldots, P_{n}\right) \stackrel{R}{\leftarrow} \mathbb{G}_{1} \times \mathbb{G}_{2} \times \cdots \times \mathbb{G}_{n} \\
\left(P_{1}, P_{2}, \ldots, P_{n}, Q\right), \text { s.t. } Q \leftarrow\left[a_{1}\right] P_{1}+\cdots+\left[a_{n}\right] P_{n} \\
\text { where }\left(a_{1}, \ldots, a_{n}\right) \stackrel{R}{\longleftarrow} \llbracket 0, A-1 \rrbracket^{n}
\end{array}\right\}
$$

It is worth mentioning that the use of these product groups in cryptography is not interesting since even if their order is $p^{n}$, the complexity of discrete logarithm computation in them is not much harder than in cyclic groups of order $p$. We will only use them as a tool in order to prove our Theorem 1 .

Following Shoup's technique [17], it is easy to prove that a generic algorithm in the product group $\mathbb{G}_{1} \times \cdots \times \mathbb{G}_{n}$ (or equivalently in $\mathbb{Z}_{p}^{n}$ ) has a negligible advantage in distinguishing the two distributions $\mathcal{U}_{n}^{\prime}$ and $\mathcal{K}_{n, A}^{\prime}$ if it makes a polynomial number of group oracle queries. More precisely, we can prove the following proposition: 
Proposition 1. Let $\mathcal{A}$ be a generic algorithm that distinguishes the two distributions $\mathcal{U}^{\prime}{ }_{n}$ and $\mathcal{K}_{n, A}^{\prime}$ and makes at most $\tau$ group oracle queries, then $\mathcal{A}$ 's advantage in distinguishing the two distributions is upper-bounded by $O\left(\tau^{2} / A^{n}\right)$.

Proof. We consider an algorithm $\mathcal{B}$ playing the following game with $\mathcal{A}$. Algorithm $\mathcal{B}$ chooses $n+1$ bit strings $\sigma_{1}, \ldots, \sigma_{n}, \sigma_{n+1}$ uniformly in $\{0,1\}^{m}$. Internally, $\mathcal{B}$ keeps track of the encoded elements using elements in the ring $\mathbb{Z}_{p}\left[X_{1}\right] \times \cdots \times \mathbb{Z}_{p}\left[X_{n}\right]$. To maintain consistency with the bit strings given to $\mathcal{A}, \mathcal{B}$ creates a lists $\mathcal{L}$ of pairs $(F, \sigma)$ where $F$ is a polynomial vector in the ring $\mathbb{Z}_{p}\left[X_{1}\right] \times \cdots \times \mathbb{Z}_{p}\left[X_{n}\right]$ and $\sigma \in\{0,1\}^{m}$ is the encoding of a group element. The polynomial vector $F$ represents the exponent of the encoded element in the group $\mathbb{G}_{1} \times \cdots \times \mathbb{G}_{n}$. Initially, $\mathcal{L}$ is set to

$$
\left\{\left((1,0, \ldots, 0), \sigma_{1}\right),\left((0,1, \ldots, 0), \sigma_{2}\right), \ldots,\left((0,0, \ldots, 1), \sigma_{n}\right),\left(\left(X_{1}, \ldots, X_{n}\right), \sigma_{n+1}\right)\right\}
$$

Algorithm $\mathcal{B}$ starts the game providing $\mathcal{A}$ with $\sigma_{1}, \ldots, \sigma_{n}, \sigma_{n+1}$. The simulation of the group operations oracle goes as follows:

Group operation: Given two encodings $\sigma_{i}$ and $\sigma_{j}$ in $\mathcal{L}, \mathcal{B}$ recovers the corresponding vectors $F_{i}$ and $F_{j}$ and computes $F_{i}+F_{j}$ (or $F_{i}-F_{j}$ ) termwise. If $F_{i}+F_{j}$ (or $F_{i}-F_{j}$ ) is already in $\mathcal{L}, \mathcal{B}$ returns to $\mathcal{A}$ the corresponding bit string; otherwise it returns a uniform element $\sigma \stackrel{R}{\leftarrow}\{0,1\}^{m}$ and stores $\left(F_{i}+F_{j}, \sigma\right)\left(\right.$ or $\left.\left(F_{i}-F_{j}, \sigma\right)\right)$ in $\mathcal{L}$.

After $\mathcal{A}$ queried the oracles, it outputs a bit $b$. At this point, $\mathcal{B}$ chooses a random bit $b^{*} \in\{0,1\}$ and uniform values $x_{1}, \ldots, x_{n} \in \mathbb{Z}_{p}$ if $b^{*}=0$ or uniform values $x_{1}, \ldots, x_{n} \in \llbracket 0, A-1 \rrbracket$ if $b^{*}=1$. The algorithm $\mathcal{B}$ sets $X_{i}=x_{i}$ for $i \in\{1, \ldots, n\}$.

If the simulation provided by $\mathcal{B}$ is consistent, it reveals nothing about $b$. This means that the probability of $\mathcal{A}$ guessing the correct value for $b^{*}$ is $1 / 2$. The only way in which the simulation could be inconsistent is if, after we choose value for $x_{1}, \ldots, x_{n}$, two different polynomial vectors in $\mathcal{L}$ happen to produce the same value. First, note that $\mathcal{A}$ is unable to cause such a collision on its own. Indeed, notice that $\mathcal{L}$ is initially populated with polynomials of degree at most one in each coordinate and that both the group addition and subtraction oracle do not increase the degree of the polynomial. Thus, all polynomials contained in $\mathcal{L}$ have degree at most one. This is enough to conclude that $\mathcal{A}$ cannot purposely produce a collision.

It remains to prove that the probability of a collision happening due to a unlucky choice of values is negligible. In other words, we have to bound the probability that two distinct $F_{i}, F_{j}$ in $\mathcal{L}$ evaluate to the same value after the substitution, namely $F_{i}\left(x_{1}, \ldots, x_{n}\right)-$ $F_{j}\left(x_{1}, \ldots, x_{n}\right)=0$. This reduces to bound the probability of hitting a zero of $F_{i}-F_{j}$. By the simulation, this happens only if $F_{i}-F_{j}$ is a non-constant polynomial vector and in this case, each coordinate is a degree one polynomial in one $X_{i}$ 's.

Recall that the Schwartz-Zippel lemma says that, if $F$ is a degree $d$ polynomial in $\mathbb{Z}_{p}\left[X_{1}, \ldots, X_{n}\right]$ and $S \subseteq \mathbb{Z}_{p}$ then

$$
\operatorname{Pr}\left[F\left(x_{1}, \ldots, x_{n}\right)=0\right] \leq \frac{d}{|S|}
$$


where $x_{1}, \ldots, x_{n}$ are chosen uniformly from $S$. Going back to our case, we obtain by applying the Schwartz-Zippel lemma to each coordinate:

$$
\operatorname{Pr}\left[\left(F_{i}-F_{j}\right)\left(x_{1}, \ldots, x_{n}\right)=\mathbf{0} \in \mathbb{Z}_{p}^{n}\right] \leq\left\{\begin{array}{c}
1 / p^{n} \text { if } b^{*}=0 \\
1 / A^{n} \text { if } b^{*}=1
\end{array}\right.
$$

Therefore, the probability that the simulation provided by $\mathcal{B}$ is inconsistent is upperbounded by $\tau(\tau-1) / A^{n}$.

We will now prove that, provided $m$ is large enough, a generic algorithm is not able to decide whether it is given as inputs $n$ generators $\left(P_{1}, \ldots, P_{n}\right)$ in a cyclic group $\mathbb{G}$ of prime order $p$ or $n$ order- $p$ elements

$$
\left(P_{1}, 1_{\mathbb{G}_{2}}, \ldots, 1_{\mathbb{G}_{n}}\right),\left(1_{\mathbb{G}_{1}}, P_{2}, \ldots, 1_{\mathbb{G}_{n}}\right), \ldots,\left(1_{\mathbb{G}_{1}}, 1_{\mathbb{G}_{2}}, \ldots, P_{n}\right)
$$

in a product group $\mathbb{G}_{1} \times \cdots \times \mathbb{G}_{n}$ where each $\mathbb{G}_{i}$ is cyclic group of prime order $p$. Note that the groups $\mathbb{G}$ and $\mathbb{G}_{1} \times \cdots \times \mathbb{G}_{n}$ are not of the same order and in practice, it will probably be easy to distinguish them. We only claim that this is difficult for a generic algorithm.

Proposition 2. Let $\mathcal{A}$ be a generic algorithm that distinguishes these two settings and makes at most $\tau$ group oracle queries, then $\mathcal{A}$ 's advantage in distinguishing the two distributions is upper-bounded by $O\left(\tau^{2} / p\right)$.

Proof. We consider an algorithm $\mathcal{B}$ playing the following game with $\mathcal{A}$. Algorithm $\mathcal{B}$ chooses a random bit $b^{*}$ and runs one of the following simulation depending on the bit $b^{*}$

- If $b^{*}=0, \mathcal{B}$ chooses $n$ bit strings $\sigma_{1}, \ldots, \sigma_{n}$ uniformly in $\{0,1\}^{m}$. Internally, $\mathcal{B}$ keeps track of the encoded elements using elements in the ring $\mathbb{Z}_{p}\left[X_{1}, \ldots, X_{n}\right]$. To maintain consistency with the bit strings given to $\mathcal{A}, \mathcal{B}$ creates a lists $\mathcal{L}$ of pairs $(F, \sigma)$ where $F$ is a polynomial in the ring $\mathbb{Z}_{p}\left[X_{1}, \ldots, X_{n}\right]$ and $\sigma \in\{0,1\}^{m}$ is the encoding of a group element. The polynomial $F$ represents the exponent of the encoded element in the group $\mathbb{G}$. Initially, $\mathcal{L}$ is set to

$$
\left\{\left(X_{1}, \sigma_{1}\right),\left(X_{2}, \sigma_{2}\right), \ldots,\left(X_{n}, \sigma_{n}\right)\right\}
$$

- If $b^{*}=1, \mathcal{B}$ chooses also $n$ bit strings $\sigma_{1}, \ldots, \sigma_{n}$ uniformly in $\{0,1\}^{m}$. Internally, $\mathcal{B}$ keeps track of the encoded elements using elements in the ring $\mathbb{Z}_{p}\left[X_{1}\right] \times \cdots \times$ $\mathbb{Z}_{p}\left[X_{n}\right]$. To maintain consistency with the bit strings given to $\mathcal{A}, \mathcal{B}$ creates a lists $\mathcal{L}$ of pairs $(F, \sigma)$ where $F$ is a polynomial vector in the ring $\mathbb{Z}_{p}\left[X_{1}\right] \times \cdots \times \mathbb{Z}_{p}\left[X_{n}\right]$ and $\sigma \in\{0,1\}^{m}$ is the encoding of a group element. The polynomial vector $F$ represents the exponent of the encoded element in the group $\mathbb{G}_{1} \times \cdots \times \mathbb{G}_{n}$. Initially, $\mathcal{L}$ is set to

$$
\left\{\left(\left(X_{1}, 0,0, \ldots, 0\right), \sigma_{1}\right),\left(\left(0, X_{2}, 0, \ldots, 0\right), \sigma_{2}\right), \ldots,\left(\left(0,0, \ldots, 0, X_{n}\right), \sigma_{n}\right)\right\}
$$

In each cases, algorithm $\mathcal{B}$ starts the game providing $\mathcal{A}$ with $\sigma_{1}, \ldots, \sigma_{n}$. The simulation of the group operations oracle goes as follows: 
Group operation: Given two encodings $\sigma_{i}$ and $\sigma_{j}$ in $\mathcal{L}, \mathcal{B}$ recovers the corresponding polynomials (or polynomial vectors, depending on $b^{*}$ ) $F_{i}$ and $F_{j}$ and computes $F_{i}+F_{j}$ (or $F_{i}-F_{j}$ ) termwise. If $F_{i}+F_{j}$ (or $F_{i}-F_{j}$ ) is already in $\mathcal{L}, \mathcal{B}$ returns to $\mathcal{A}$ the corresponding bit string; otherwise it returns a uniform element $\sigma \stackrel{R}{\longleftarrow}\{0,1\}^{m}$ and stores $\left(F_{i}+F_{j}, \sigma\right)\left(\right.$ or $\left.\left(F_{i}-F_{j}, \sigma\right)\right)$ in $\mathcal{L}$.

After $\mathcal{A}$ queried the oracles, it outputs a bit $b$. At this point, $\mathcal{B}$ chooses uniform values $x_{1}, \ldots, x_{n} \in \mathbb{Z}_{p}$. The algorithm $\mathcal{B}$ sets $X_{i}=x_{i}$ for $i \in\{1, \ldots, n\}$.

If the simulation provided by $\mathcal{B}$ is consistent, it reveals nothing about $b$. This means that the probability of $\mathcal{A}$ guessing the correct value for $b^{*}$ is $1 / 2$. The only way in which the simulation could be inconsistent is if, after we choose value for $x_{1}, \ldots, x_{n}$, two different polynomial vectors in $\mathcal{L}$ happen to produce the same value. First, note that $\mathcal{A}$ is unable to cause such a collision on its own. Indeed, notice that $\mathcal{L}$ is initially populated with polynomials of degree at most one in each coordinate and that both the group addition and subtraction oracle do not increase the degree of the polynomial. Thus, all polynomials contained in $\mathcal{L}$ have degree at most one. This is enough to conclude that $\mathcal{A}$ cannot purposely produce a collision.

It remains to prove that the probability of a collision happening due to a unlucky choice of values is negligible. If $b^{*}=1$, the probability of a collision happening is equal to 0 . If $b^{*}=0$, we have to bound the probability that two distinct $F_{i}, F_{j}$ in $\mathcal{L}$ evaluate to the same value after the substitution, namely $F_{i}\left(x_{1}, \ldots, x_{n}\right)-F_{j}\left(x_{1}, \ldots, x_{n}\right)=0$. This reduces to bound the probability of hitting a zero of $F_{i}-F_{j}$.

Applying the Schwartz-Zippel lemma, we obtain

$$
\operatorname{Pr}\left[\left(F_{i}-F_{j}\right)\left(x_{1}, \ldots, x_{n}\right)=0 \in \mathbb{Z}_{p}\right] \leq 1 / p
$$

Therefore, the probability that the simulation provided by $\mathcal{B}$ is inconsistent is upperbounded by $\tau(\tau-1) / p$.

To prove Theorem 1 , it is then enough to prove that if there exists a generic algorithm that distinguishes the two distributions $\mathcal{U}_{n}$ and $\mathcal{K}_{n, A}$ that makes at most $\tau$ group oracle queries with an advantage larger than $\Omega\left(\tau^{2} / A^{n}\right)$, it gives an adversary able to distinguish the cyclic group setting from the product group setting making at most $\tau$ group oracle queries and with advantage $\Omega\left(\tau^{2} / A^{n}\right)$ (due to Proposition 11) and this result contradicts Proposition 2

\subsection{Description of Our Protocol}

In the previous subsection, we provided a description of a basic version of our protocol. In this subsection, we consider an improved version of it on elliptic curves equipped with efficient endomorphisms. In this improved scheme, instead of masking $\mathcal{S} \mathcal{K}$ with $\left[a_{1}\right] P_{1}+\cdots+\left[a_{n-1}\right] P_{n-1}$ with $\left(a_{1}, \ldots, a_{n-1}\right) \stackrel{R}{\longleftarrow} \llbracket 0, A-1 \rrbracket^{n-1}$, we will masked it with $\left[a_{1}\right] Q_{1}+\cdots+\left[a_{n-1}\right] Q_{n-1}$ with $\left(a_{1}, \ldots, a_{n-1}\right) \stackrel{R}{\longleftarrow} \llbracket 0, A-1 \rrbracket^{n-1}$ where the $Q_{i}$ 's are images of the $P_{i}$ under one of the efficient endomorphisms defined on the curve. If we denote $\mathcal{S}$ the set of efficient endomorphisms on the curve (that can also be efficiently evaluated in the group $\mathbb{G}_{T}$ ), we obtained a scheme with generic security $\Omega\left(\# \mathcal{S}^{n-1} \cdot A^{n-1 / 2}\right)$. 
Setup (could be offline). In the following, the smartcard has to generate several random points on an elliptic curve $E\left(\mathbb{F}_{p}\right)$. Fouque and Tibouchi [11] proposed an efficient method to do it on a BN curve.

1. Let $\mathcal{I}$ a set of small integers, $\mathcal{I}=\left\{0,1,2,3,4,5, \ldots, 2^{\ell}-1\right\}$ with $\# \mathcal{I}=2^{\ell}=A$.

2. The smartcard generates $n-1$ random points $P_{1}, P_{2}, \ldots, P_{n-1}$ on the elliptic curve $E\left(\mathbb{F}_{p}\right)$.

3. The smartcard chooses an endomorphism $\sigma_{i} \in \mathcal{S}$ to apply to $P_{i}$ and sets $Q_{i}=$ $\sigma_{i}\left(P_{i}\right)$.

4. For each point $Q_{i}$, the smartcard takes at random $\alpha_{i} \in \mathcal{I}$ and sets

$$
P_{n}=\mathcal{S K}-\left(\left[\alpha_{1}\right] Q_{1}+\left[\alpha_{2}\right] Q_{2}+\ldots+\left[\alpha_{n-1}\right] Q_{n-1}=\mathcal{S K}-\sum_{i=1}^{n-1}\left[\alpha_{i}\right] Q_{i} .\right.
$$

\section{Delegation}

5. The smartcard sends $P_{1}, P_{2}, \ldots, P_{n}$ to the server.

6. The server computes the $n$ pairings $f_{i}=e\left(Q_{i}, \mathcal{P} \mathcal{P}\right)$ and sends them back to the smartcard.

\section{Session key computation}

7. The smartcard computes $\left(f_{1}^{\sigma_{1}}\right)^{\alpha_{1}} \cdot\left(f_{2}^{\sigma_{2}}\right)^{\alpha_{2}} \cdots\left(f_{n-1}^{\sigma_{n-1}}\right)^{\alpha_{n-1}} \cdot f_{n}=\mathcal{K}$. The $\sigma_{i}$ are also almost free. Thanks to the bilinearity property,

$$
\begin{aligned}
e(\mathcal{S K}, \mathcal{P} \mathcal{P}) & =e\left(\alpha_{1} Q_{1}+\alpha_{2} Q_{2} \ldots+\alpha_{n-1} Q_{n-1}+P_{n}, \mathcal{P} \mathcal{P}\right) \\
& =e\left(\alpha_{1} Q_{1}, \mathcal{P} \mathcal{P}\right) e\left(\alpha_{2} Q_{2}, \mathcal{P} \mathcal{P}\right) \cdots e\left(\alpha_{n-1} Q_{n-1}, \mathcal{P} \mathcal{P}\right) e\left(P_{n}, \mathcal{P P}\right) \\
& =\left(e\left(P_{1}, \mathcal{P} \mathcal{P}\right)^{\sigma_{1}}\right)^{\alpha_{1}} \cdots\left(e\left(P_{n-1}, \mathcal{P} \mathcal{P}\right)^{\sigma_{n-1}}\right)^{\alpha_{n-1}}\left(e\left(P_{n}, \mathcal{P P}\right)\right)
\end{aligned}
$$

with $\sigma_{i}$ a cheap endomorphism in $\mathbb{F}_{p^{k}}^{*}$ such that $e\left(\sigma_{i}\left(P_{i}\right), \mathcal{P} \mathcal{P}\right)=e\left(P_{i}, \mathcal{P} \mathcal{P}\right)^{\sigma_{i}}$.

Example on on a Barreto-Naehrig curve. For optimal Ate pairing on a BN curve with 128-bit security level (i.e. 256-bit prime number $p$ ), the endomorphism set $\mathcal{S}$ can be defined as $\left\{\mathrm{Id},-\mathrm{Id}, \phi, \phi^{2},-\phi,-\phi^{2}\right\}$ where $\phi$ is computed from the complex multiplication endomorphism available on the curve. These endomorphisms are almost free on $E\left(\mathbb{F}_{p}\right)$ if $D=1$ or $D=3$. They cost at most one multiplication and one subtraction in $\mathbb{F}_{p}$ and the resulting point $Q_{i}$ is still in affine coordinates [5].

In the Setup procedure, the smartcard has to obtain $P_{n}$ in affine coordinates, this costs one inversion in $\mathbb{F}_{p}$ plus four multiplications, resulting in an additional cost of (say) $64 M_{p}$. The cost of computing $P_{n}$ is $(n-1) \cdot(\ell \cdot 7+\ell / 2 \cdot 11+16)+64 M_{p}$. Indeed, in Jacobian coordinates, one addition on $E\left(\mathbb{F}_{p}\right)$ with one of the two points in affine coordinates costs $11 M_{p}$, if none of the points are in affine coordinates, this costs $16 M_{p}$, and one doubling costs $8 M_{p}$. If moreover we use a BN curve $\left(a_{4}=0\right)$, a doubling costs $7 M_{p}$.

The computation cost for the powerful device is $16336(0.84(n-1)+1) M_{p}$. Indeed, the first pairing costs $\approx 16336 M_{p}$ and the $(n-1)$ other ones cost 0.84 of one 


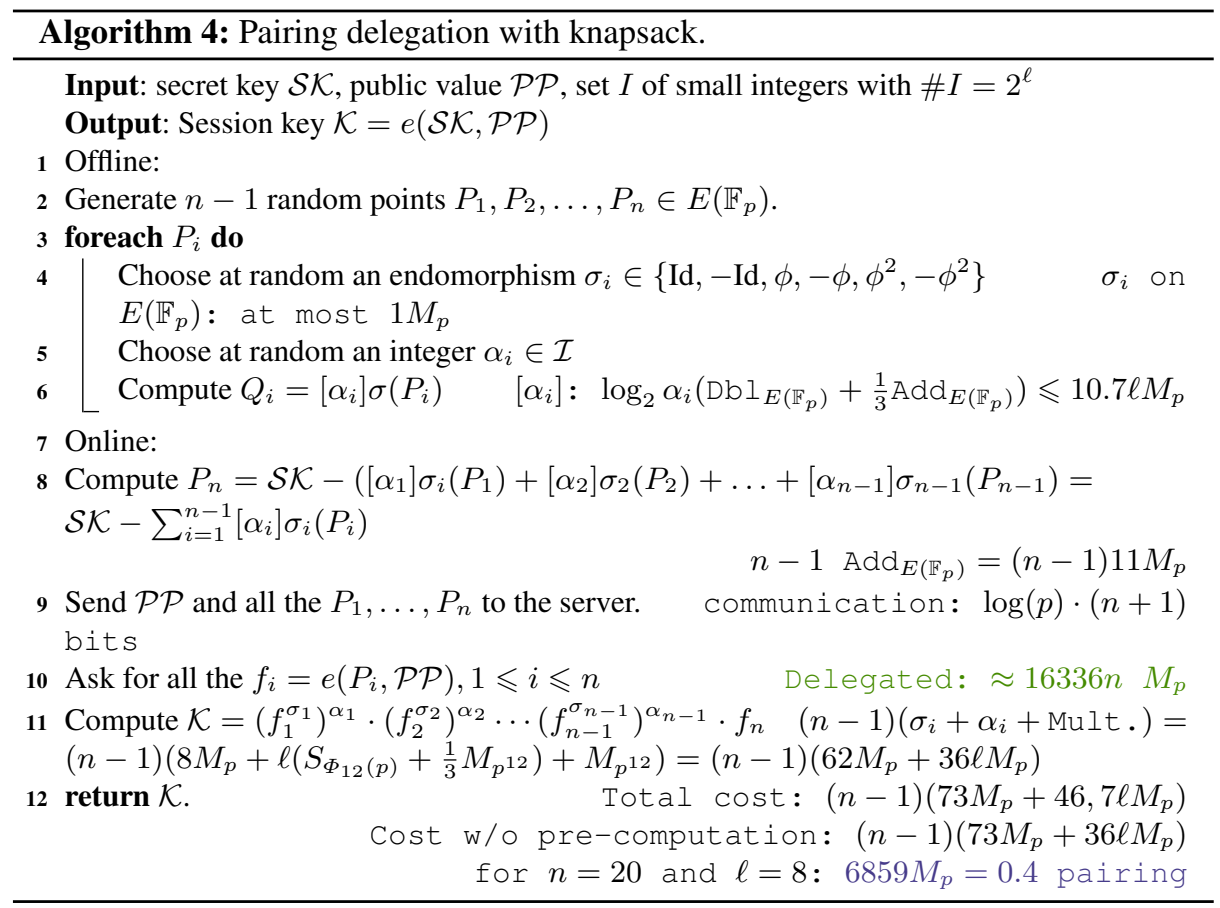

pairing (since the second argument is the fixed point $\mathcal{P} \mathcal{P}$, the tangents and lines can be computed from $\mathcal{P} \mathcal{P}$ one single time for all the pairings).

Finally, the smartcard computes $n-1$ exponentiations and multiplies $n$ elements in $\mathbb{G}_{T}$ to obtain the session key $\mathcal{K}=e(\mathcal{S K}, \mathcal{P} \mathcal{P})$. An exponentiation costs in average $\ell$ squaring plus $\ell / 2$ multiplications in $\mathbb{F}_{p^{12}}$. The $n-1$ exponentiations cost $(n-1)(18 \ell+$ $54 \ell / 3) M_{p}$. It remains to compute $n-1$ multiplications.

Overall, we obtain the global cost for the restricted device is: $(n-1)\left(73 M_{p}+\right.$ $\left.46,7 \ell M_{p}\right)$ (and $(n-1)\left(73 M_{p}+36 \ell M_{p}\right)$ is pre-computation is possible). We summarize our proposition in Alg. 4. By choosing appropriate values for $n$ and $\ell$, one can achieve various communication-efficiency trade-off as shown in Tab. 2. To achieve statistical security (instead of generic computational security), one basically needs to double the value of $\ell$. One can find parameters for which the delegation procedure is more efficient than the pairing computation ( 0.5 pairing for practical parameters).

\section{Partial Pairing Computation Delegation}

In this final section, we propose a completely different approach based on the arithmetic of the pairing computation (without verifiability) of a pairing $e(\mathcal{S K}, \mathcal{P} \mathcal{P})$ of a secret key $\mathcal{S} \mathcal{K}$ and some public parameter $\mathcal{P} \mathcal{P}$. More precisely, we delegate only the non-critical

\footnotetext{
${ }^{1}$ It is worth mentioning that this computational cost can be further decreased by using classical multi-exponentiation techniques (in particular for small values of $n($ e.g. $n=5$ ).
} 
Table 2. Communication/Efficiency Trade-off of our knapsack delegation protocol

\begin{tabular}{|c|c|c|c|c|}
\hline$\ell$ & $n$ & Generic Security & Computational Cost & Communication \\
\hline 59 & 5 & 128 & $8788 M_{p}=0.53$ pairing & 15360 bits \\
\hline 23 & 10 & 126 & $8109 M_{p}=0.49$ pairing & 30720 bits \\
\hline 13 & 15 & 127 & $7574 M_{p}=0.46$ pairing & 46080 bits \\
\hline 8 & 20 & 125 & $6859 M_{p}=0.41$ pairing & 61440 bits \\
\hline 8 & 20 & 125 & $6859 M_{p}=0.41$ pairing & 61440 bits \\
\hline 5 & 25 & 122 & $6072 M_{p}=0.37$ pairing & 76800 bits \\
\hline 3 & 30 & 118 & $5249 M_{p}=0.32$ pairing & 92160 bits \\
\hline 0 & 51 & 128 & $3650 M_{p}=0.22$ pairing & 156672 bits \\
\hline
\end{tabular}

steps in the pairing algorithm, looking carefully at each instruction in Miller algorithm. The protocol only achieves weak secrecy: the helper will learn the session key $\mathcal{K}$ (but still not the secret key $\mathcal{S} \mathcal{K}$ ).

Final Powering Delegation We can blind the output $f^{\prime} \leftarrow u \cdot f$ of the Miller function by an element $u \in \mathbb{F}_{p^{k}}^{*}$ which is an $r$-th power (there exists a $u^{\prime} \in \mathbb{F}_{p^{k}}^{*}$ such that $u^{\prime}=u$ ), see Alg. 5. Hence $u$ will disappear after the final powering $f^{\frac{p^{k}-1}{r}}$ (Alg. 11 lines 16 27) since $u^{\frac{p^{k}-1}{r}}=u^{\prime p^{k}-1}=1$. So we can delegate the final powering thanks to the equality $f^{\prime\left(p^{k}-1\right) / r}=(u f)^{\left(p^{k}-1\right) / r}=\mathcal{K}$ the session key. The helper learns the session key $\mathcal{K}$ but as no additional information on $f$ (in particular pairing inversion is not possible).

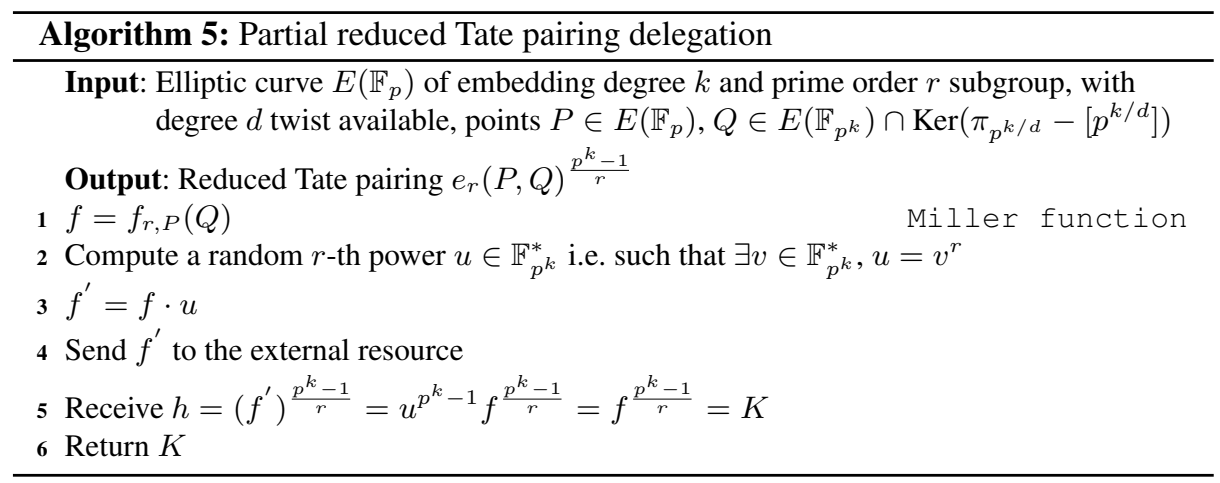

Tangent and line Delegation The two points $P, Q$ play two different roles in a Tate-like pairing computation. In an ate pairing, the point $P$ is used to evaluate the intermediate line functions $\ell(P)$. The line functions $\ell$ are computed through a scalar multiplication $[s] Q$ (with $s$ a public parameter of the curve). The coefficients arising in the lines and tangent computation are re-used to update the Miller function $f_{s, \mathcal{P} P}(\mathcal{S K})$. If $Q$ is actually a public parameter $\mathcal{P} \mathcal{P}$, then the line computation $\ell_{\mathcal{P} \mathcal{P}}$ can be delegated. The 
restricted device (such as a smartcard) will ask for the successive intermediate values $\ell$ then evaluate them at the secret point $P=\mathcal{S K}$.

For an ate pairing on a BN curve, the line is of the form $\ell=\ell_{0}+\ell_{1} \omega+\ell_{3} \omega^{3}$, with $\mathbb{F}_{p^{12}}=\mathbb{F}_{p^{2}}[\omega]=\mathbb{F}_{p^{2}}[\omega] /\left(\omega^{6}-\xi\right)$. The smartcard can delegate the computation of the three coefficients then compute the line equation evaluated at $\mathcal{S K}$.

Tangent and line computation One can found Relic [1] formulas for tangent and line computation in src/pp/relic_pp_dbl . c (function pp_dbl_k12_projc_basic) and src/pp/relic_pp_add.c (function pp_add_k12_projc_basic).

We recall the formula from [2, eq. (10)]:

$$
\ell_{2 T}(P)=-2 Y Z y_{P}+3 X^{2} x_{P} \omega+\left(3 b^{\prime} Z^{2}-Y^{2}\right) \omega^{3}
$$

with $\omega$ such that $\mathbb{F}_{p^{12}}=\mathbb{F}_{p^{2}}[\omega] /\left(\omega^{6}-\xi\right), X, Y, Z \in \mathbb{F}_{p^{2}}$ and $x_{P}, y_{P} \in \mathbb{F}_{p}$.

The second formula is the following [2, eq. (13)], with $L=X-x_{Q} Z$ and $M=$ $Y-y_{Q} Z$ :

$$
\ell_{T+Q}(P)=-L y_{P}-M x_{P} \omega+(M X-L Y) \omega^{3}
$$

In both cases the coefficients of $\ell$ are computed from a public parameter $Q=\mathcal{P} \mathcal{P}$ hence can be delegated. The smart card saves $2 S_{p^{2}}+7 M_{p^{2}}=$ It remains for the smart card to evaluate the line $\ell$ at $\mathcal{S K}=P=\left(x_{P}, y_{P}\right)$. This costs $4 M_{p}$ in both cases.

Efficiency improvement. To sum up, the smartcard sends the point $\mathcal{P} \mathcal{P}$ to the external computer and computes the intermediate values of the Miller function on the fly, when receiving the coefficients of the intermediate values. No information on $\mathcal{S K}$ is provided to the external helper (except $f^{\prime}$ which does not reveal more information than the session key $\mathcal{K}$ ). For an optimal Ate pairing on a Barreto-Naehrig curve, this saves $31 \%$ of the Miller loop, then we delegate $100 \%$ of the final powering, saving at the end $65 \%$ of the pairing cost. Note that the idea can be adapted to achieve (strong) secrecy by further masking the final powering but the efficiency improvement is smaller if pre-computation is not possible. Note also that the same idea can be applied to any instantiation of pairings (but requires a specific analysis).

Acknowledgements. The authors thank Olivier Blazy, Renaud Dubois and Fabien Laguillaumie for their fruitful comments. This work was supported in part by the French ANR-12-INSE-0014 SIMPATIC Project.

\section{References}

1. D. F. Aranha and C. P. L. Gouvêa. RELIC is an Efficient LIbrary for Cryptography. http: //code.google.com/p/relic-toolkit/, September 2013.

2. Diego F. Aranha, Paulo S. L. M. Barreto, Patrick Longa, and Jefferson E. Ricardini. The realm of the pairings. In SAC 2013: 20th Annual International Workshop on Selected Areas in Cryptography, Lecture Notes in Computer Science, pages 3-25. Springer, 2013.

3. Paulo S. L. M. Barreto and Michael Naehrig. Pairing-friendly elliptic curves of prime order. In Bart Preneel and Stafford Tavares, editors, SAC 2005: 12th Annual International Workshop on Selected Areas in Cryptography, volume 3897 of Lecture Notes in Computer Science, pages 319-331. Springer, August 2005. 
4. Dan Boneh and Matthew K. Franklin. Identity-based encryption from the Weil pairing. In Joe Kilian, editor, Advances in Cryptology - CRYPTO 2001, volume 2139 of Lecture Notes in Computer Science, pages 213-229. Springer, August 2001.

5. Joppe W. Bos, Craig Costello, and Michael Naehrig. Exponentiating in pairing groups. Cryptology ePrint Archive, Report 2013/458, 2013.

6. Victor Boyko, Marcus Peinado, and Ramarathnam Venkatesan. Speeding up discrete log and factoring based schemes via precomputations. In Kaisa Nyberg, editor, Advances in Cryptology - EUROCRYPT'98, volume 1403 of Lecture Notes in Computer Science, pages 221-235. Springer, May / June 1998.

7. Sébastien Canard, Julien Devigne, and Olivier Sanders. Delegating a pairing can be both secure and efficient. In ACNS 14: 12th International Conference on Applied Cryptography and Network Security, Lecture Notes in Computer Science, pages 549-565. Springer, 2014.

8. Benoit Chevallier-Mames, Jean-Sebastien Coron, Noel McCullagh, David Naccache, and Michael Scott. Secure delegation of elliptic-curve pairing. Cryptology ePrint Archive, Report 2005/150, 2005.

9. - - Secure delegation of elliptic-curve pairing. In Dieter Gollmann, Jean-Louis Lanet, and Julien Iguchi-Cartigny, editors, Smart Card Research and Advanced Application, 9th IFIP WG 8.8/11.2 International Conference, CARDIS 2010, volume 6035 of Lecture Notes in Computer Science, pages 24-35. Springer, 2010.

10. Jean-Sébastien Coron, David M'Raïhi, and Christophe Tymen. Fast generation of pairs $(k,[k] P)$ for Koblitz elliptic curves. In Serge Vaudenay and Amr M. Youssef, editors, SAC 2001: 8th Annual International Workshop on Selected Areas in Cryptography, volume 2259 of Lecture Notes in Computer Science, pages 151-164. Springer, August 2001.

11. Pierre-Alain Fouque and Mehdi Tibouchi. Indifferentiable hashing to Barreto-Naehrig curves. In Alejandro Hevia and Gregory Neven, editors, Progress in Cryptology - LATINCRYPT 2012: 2nd International Conference on Cryptology and Information Security in Latin America, volume 7533 of Lecture Notes in Computer Science, pages 1-17. Springer, October 2012.

12. Marc Girault and David Lefranc. Server-aided verification: Theory and practice. In Bimal K. Roy, editor, Advances in Cryptology - ASIACRYPT 2005, volume 3788 of Lecture Notes in Computer Science, pages 605-623. Springer, December 2005.

13. Robert Granger and Michael Scott. Faster squaring in the cyclotomic subgroup of sixth degree extensions. In Phong Q. Nguyen and David Pointcheval, editors, PKC 2010: 13th International Conference on Theory and Practice of Public Key Cryptography, volume 6056 of Lecture Notes in Computer Science, pages 209-223. Springer, May 2010.

14. Antoine Joux. A one round protocol for tripartite diffie-hellman. In Wieb Bosma, editor, ANTS, volume 1838 of Lecture Notes in Computer Science, pages 385-394. Springer, 2000.

15. Ezekiel J. Kachisa, Edward F. Schaefer, and Michael Scott. Constructing Brezing-Weng pairing-friendly elliptic curves using elements in the cyclotomic field. In Steven D. Galbraith and Kenneth G. Paterson, editors, PAIRING 2008: 2nd International Conference on Pairingbased Cryptography, volume 5209 of Lecture Notes in Computer Science, pages 126-135. Springer, September 2008.

16. Phong Q. Nguyen, Igor E. Shparlinski, and Jacques Stern. Distribution of modular sums and the security of the server aided exponentiation. In Cryptography and Computational Number Theory, volume 20 of Progress in Computer Science and Applied Logic, pages 331342. Springer, 2001.

17. Victor Shoup. Lower bounds for discrete logarithms and related problems. In Walter Fumy, editor, Advances in Cryptology - EUROCRYPT'97, volume 1233 of Lecture Notes in Computer Science, pages 256-266. Springer, May 1997. 